\title{
Noma Neonatorum A Review of Unusual Presentation of Pseudomonas Sepsis in Neonates
}

\author{
Santosh Kumar Kamalakannan* \\ Department of Neonatology, Saveetha Medical College and Hospital, India \\ *Corresponding author: Santosh Kumar Kamalakannan, Department of Neonatology, Saveetha medical college and hospital, \\ Thandalam, Chennai, India
}

\begin{abstract}
Noma Neonatorum is a gangrenous infection involving the mucocutaneous junctions of oral, nasal,anal area and rarely the eyelids . It generally affects the premature low birth weight babies in the first few weeks of neonatal life. This rare complication of Pseudomonas septicemia has a very high fatality documented in previous literature.
\end{abstract}

Keywords: Noma Neonatorum; Pseudomonas Spp; Facial Palsy; Neonatal Sepsis

\section{Case Report}

A36 weeks male baby weighing 2400 grams, with an uneventful antenatal and perinatal period was referred from nearby health care facility for a bluish erythematous macular lesion over face bear the nose which was noticed on day 3 of life .Careful enquiry of the history and events revealed that the neonate did not require any form of resuscitation at birth and was not previously admitted in any hospital prior to the onset of this lesion .Examination of the neonate revealed a sick neonate with a necrotizing lesion which involved the right ala nasi and there was substantial perilesional edema .Careful examination also demonstrated that the neonate was having a left sided Lower motor neuron type of facial nerve palsy and there was ipsilateral purulent ear discharge. Other examination was essentially normal. A working diagnosis of Neonatal sepsis with probability of Pseudomonas infection (Ecthyma Gangrenosa was made and child was started on AntiPseudomonal antibiotics.

Cultures were sent from the wound, ear discharge and blood,which grew Pseudomonas organism which was sensitive to Meropenam. Surgical consultation was obtained and the wound debriment was done. Antibiotics were continued for as per the sensitivity pattern. The neonate showed remarkable improvement in his general status and there was a recovery of the facial palsy by day 7 of antibiotics. After three weeks of supportive and specific antimicrobial management child was discharged, now the is 18 months old with normal neurodevelopment outcome. The review of literature revealed similar presentation of Pseudomonas infection in neonates and the terminology Noma Neonatorum being used for the same.

\section{Discussion}

Ecthyma gangrenosum (EG) is a bacterial skin infection caused by pseudomonas aeruginosa. It commonly occurs as a part of generalized sepsis due to Pseudomanas and rarely skin involvement can be the primary focus of infection. Ecthyma usually occurs in immunocompromised individuals. The characteristic clinical presentation is an erythematous macule that develops into a hemorrhagic vesicle and finally transforms into a necrotic ulcer. It should always be kept as a differential diagnosis of ulcerous skin lesions inneonates especially preterm babies as rapid diagnosis can be lifesaving and reduces the incidence of mortality. The incidence of Ecthyma in Neonates with Pseudomaonas sepsis is between $1 \%$ and $3 \%$. Pseudomanas is also one of the leading causes of sepsis in neonates and moreso in hospitalized neonates. Ecthyma 
usually occurs in the anogenital region, the extremities and over the trunk,rarely does it occur over the face and the neck .But interestingly preterm neonates with ecthyma have involvement of the face and neck according to previous reports. This characteristic presentation probably is the basis for the orgin of the term "Noma Neonatorum" coined by Ghosal et al. [1] Because of the clinical similarity between this type of Ecthyma and Noma (cancrum oris) $[2]$.

\section{Noma And Noma Neonatorum}

Noma and noma neonatorum are actually different entities. Noma (Cancrus oris )is described in children aged between 2-5 years and is caused predominantly by Borellia vincentie, Bacillus fusiformis, Bacteroids and Staphylococcus species [1], on the other hand Noma Neonatorum is usually seen in neonates and Pseudomonas aeruginosa is the most common causative organism[3,4].There have few reports of Ecoli and Acinetobactor sepsis having similar presentation also. On review of the available literature, less the 80 such cases of Noma neonatorum have been reported hencefar [1-11]. The terminology as previously discussed was coined by Ghosal et al. who had managed 48 such neonates in Kolkata in 1970s. The first such case was probably reported in the year 1930[1,2] after which there have been reports of similar condition from China[6] ,Israel[5] ,India [7] and western countries $[1,3,8,9]$ also. Noma neonatorum has predilection for low birth birthweight and preterm neonates who have been found to be affected more compared to term neonates . However, there are reports of Term neonates also being affected. [10] Oral cavity is the most common site of involvement, the others being nose, eyelid, umbilicus, scrotum and groin. Onset of the disease has been reported to range from 3 rd postnatal day to 120 days. Pseudomonas aeruginosa has consistently been isolated from all cases. The disease is fatal in majority of the cases Freeman et al. Later included these cases of noma neonatorum in the description of Ecthyma because of their common etiologic pathogen Pseudomona which is practically inexistent in classic Noma [11]. The diagnosis of Noma Neonatorum is mainly clinical and must be confirmed by cultures taken from the lesions. Positive culture allows Noma Neonatorum to be distinguished from other types of lesions in the differential diagnosis, such as deep mycosis or lesions caused by anaerobic pathogens. The goal of treatment is to resolve the underlying bacteremia, which affects the prognosis, and should include synergistic antibiotics generally consisting of an aminoglycoside and an antipseudomonal $\beta$-lactam. The lesion should be treated with topical antibiotics and mechanical or chemical debridement.

\section{Conclusion}

Noma Neonatorum must be considered in the differential diagnosis of ulcerous lesions in preterm neonates especially when they are found over the face and neck region. Timely surgical intervention and specific antimicrobial treatment can result in excellent outcomes.

\section{References}

1. Ghosal SP, Chaudhari M, Dutta N, Sarkar AK, Mukherjee AK, et al. (1977) Noma Neonatorum. Indian Pediatr 14: 709-714.

2. Tempest MN (1996) Cancrum oris. Br J Surg 53: 949-969.

3. Eisele DW, Inglis AF Jr, Richardson MA (1990) Noma and noma neonatorum. Ear Nose Throat J 69:119-123.

4. Ghosal SP, Sen Gupta PC, Mukherjee AK, Choudhury M, Datta N, et al. (1978) Noma neonatorum: It's etiopathogenesis. Lancet 2: 289- 290.

5. Alkalay A, Mogilner BM, Nissim F, Barak Y, Handzel ZT, et al. (1985) Noma in full term neonate. Clin Pediatr (Phila) 24: 528-530.

6. Lin JY, Wang DW, Peng CT, Tsai FJ, Chiou YM, et al. (1992) Noma neonatorum: An unusual case of noma involving a full-term neonate. Acta Pediatr 81(9): 720-722.

7. Borle RM, Agarwal M (1987) Noma neonatorum. Int J Oral Maxillofac Surg 16: 626-629.

8. Griffin JM, Back DE, Nespec JA, et al. Noma Report of two cases. Oral Surg 1983,56: 605-607.

9. Adekeye EO, Ord RA (1983) Cancrum Oris: Principles of management of reconstructive surgery. J Maxillofac Surg 11(4): 160-170.

10. Atiyeh BS, Hashim HA, Rubeiz MT, Hamdan AM, Bitar FF, et al. (1998) Necrotising infection of the orofacial tissues in neonates (Noma Neonatorum). Case report. Scand J Plast Reconstr Surg Hand Surg 32(3): 343-345.

11. Freeman AF, Mancini AJ, Yogev R (2002) Is Noma Neonatorum a presentation of ecthyma gangrenosum in the newborn? Pediatr Infect Dis J 21(1): 83-85.

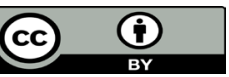

This work is licensed under Creative Commons Attribution 4.0 License

To Submit Your Article Click Here:

Submit Article

DOI: $10.32474 /$ PAPN.2020.02.000150

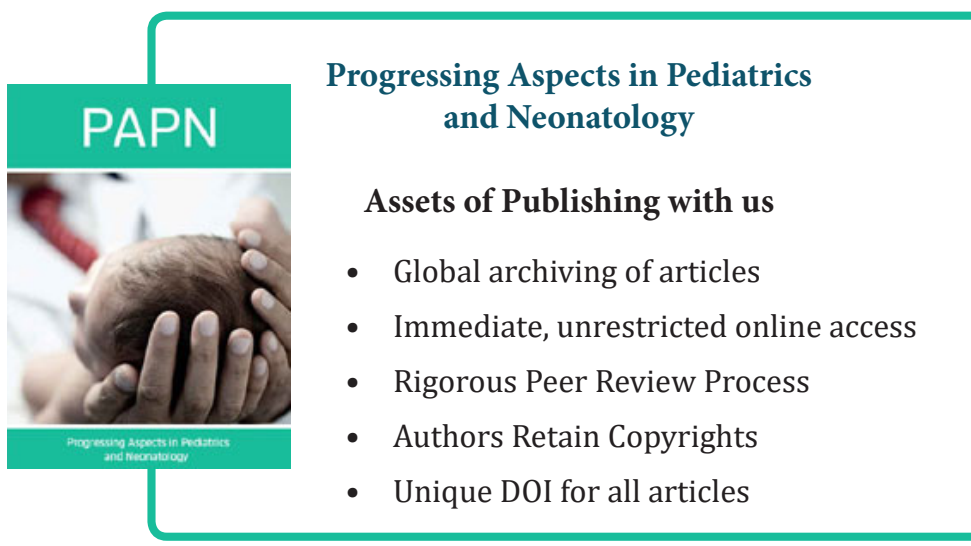

